\title{
Partial wave analysis of the Dirac fermions scattered from Reissner-Nordström charged black holes
}

\author{
Ion I. Cotaescu ${ }^{\mathrm{a}}$, Cosmin Crucean $^{\mathrm{b}}$, Ciprian A. Sporea $^{\mathrm{c}}$ (]) \\ West University of Timişoara, V. Pârvan Ave. 4, 300223 Timisoara, Romania
}

Received: 26 April 2016 / Accepted: 12 July 2016 / Published online: 22 July 2016

(C) The Author(s) 2016. This article is published with open access at Springerlink.com

\begin{abstract}
The asymptotic form of Dirac spinors in the field of the Reissner-Nordström black hole is derived for the scattering states (with $E>m c^{2}$ ) obtaining the phase shifts of the partial wave analysis of Dirac fermions scattered from charged black holes. Elastic scattering and absorption are studied giving analytic formulas for the partial amplitudes and cross sections. A graphical study is performed for analyzing the differential cross section (forward/backward scattering) and the polarization degrees as functions of the scattering angle.
\end{abstract}

\section{Introduction}

The problem of the quantum fermions scattered from Schwarzschild black holes was studied either in particular cases $[1,2]$ or by using combined analytical and numerical methods [3-7]. Recently we performed an analytic study of this process proposing a version of partial wave analysis that allowed us to write down closed formulas for the scattering amplitudes and cross sections [8]. The analytic approach improves our understanding of the quantum mechanisms that governs fermion scattering by black holes.

In the present paper we would like to extend this analytic study to the problem of the Dirac fermions scattered from Reissner-Nordström charged black holes since it seems that this problem was neglected so far. The studies performed in the existing literature have been concentrating mainly on scalar field [9-12] and electromagnetic scattering [13-16] on charged black holes. For these reasons we concentrate on studying the problem of fermion scattering on a ReissnerNordström charged black hole by using analytical and graphical methods. This phenomenon could be interesting since along with the gravitational interaction we can study the

\footnotetext{
a e-mail: cota@physics.uvt.ro

b e-mail: crucean@physics.uvt.ro

c e-mail: ciprian.sporea89@e-uvt.ro
}

effect of the interaction between the charges of the black hole and the fermion charge upon the scattering process. In addition the results related to the fermion absorption by the Schwarzschild black hole will be modified depending on the electric attraction/repulsion between the black hole charge and the fermion charge.

We deduce the asymptotic form of the Dirac spinors in the Reissner-Nordström geometry deriving the phase shifts and the partial amplitudes of elastic scattering as well as the absorption cross section. We present the principal analytic results without extended examples or comments that exceed the space of this short paper. We use the methods and notations of Ref. [8] and Planck's natural units with $G=c=\hbar=1$.

\section{Asymptotic spinors in Reissner-Nordström geometry}

The Dirac equation in curved spacetimes is defined in frames $\{x ; e\}$ formed by a local chart of coordinates $x^{\mu}$, labeled by natural indices, $\alpha, \ldots, \mu, v, \ldots=0,1,2,3$, and an orthogonal local frame and coframe defined by the gauge fields (or tetrads), $e_{\hat{\alpha}}$ and, respectively, $\hat{e}^{\hat{\alpha}}$, labeled by the local indices $\hat{\alpha}, \ldots, \hat{\mu}, \ldots$ with the same range. In local-Minkowskian manifolds $(M, g)$, having as a flat model the Minkowski spacetime $\left(M_{0}, \eta\right)$ of metric $\eta=\operatorname{diag}(1,-1,-1,-1)$, the gauge fields satisfy the usual duality conditions, $\hat{e}_{\alpha}^{\hat{\mu}} e_{\hat{v}}^{\alpha}=$ $\delta_{\hat{v}}^{\hat{\mu}}, \hat{e}_{\alpha}^{\hat{\mu}} e_{\hat{\mu}}^{\beta}=\delta_{\alpha}^{\beta}$ and the orthogonality relations, $e_{\hat{\mu}} \cdot e_{\hat{v}}=$ $\eta_{\hat{\mu} \hat{v}}, \hat{e}^{\hat{\mu}} \cdot \hat{e}^{\hat{v}}=\eta^{\hat{\mu} \hat{v}}$. The gauge fields define the 1-forms $\omega^{\hat{\mu}}=\hat{e}_{\nu}^{\hat{\mu}} \mathrm{d} x^{\nu}$ giving the line element $\mathrm{d} s^{2}=\eta_{\hat{\alpha} \hat{\beta}} \omega^{\hat{\alpha}} \omega^{\hat{\beta}}=$ $g_{\mu \nu} \mathrm{d} x^{\mu} \mathrm{d} x^{\nu}$.

Let us consider the Dirac equation, $i \gamma^{\hat{\alpha}} D_{\hat{\alpha}} \psi-m \psi=0$ of a free spinor field $\psi$ of mass $m$, written with our previous notations [8] in the frame $\{x ; e\}$ defined by the Cartesian gauge $[17,18]$,

$\omega^{0}=w(r) \mathrm{d} t$ 


$$
\begin{aligned}
\omega^{1}= & \frac{1}{w(r)} \sin \theta \cos \phi \mathrm{d} r+r \cos \theta \cos \phi \mathrm{d} \theta \\
& -r \sin \theta \sin \phi \mathrm{d} \phi, \\
\omega^{2}= & \frac{1}{w(r)} \sin \theta \sin \phi \mathrm{d} r+r \cos \theta \sin \phi \mathrm{d} \theta \\
& +r \sin \theta \cos \phi \mathrm{d} \phi, \\
\omega^{3}= & \frac{1}{w(r)} \cos \theta \mathrm{d} r-r \sin \theta \mathrm{d} \theta,
\end{aligned}
$$

in the central gravitational and Coulomb field of a charged black hole of mass $M$ and charge $Q>0$ with the ReissnerNordström line element

$$
\begin{aligned}
\mathrm{d} s^{2} & =\eta_{\hat{\alpha} \hat{\beta}} \omega^{\hat{\alpha}} \omega^{\hat{\beta}} \\
& =w(r)^{2} \mathrm{~d} t^{2}-\frac{\mathrm{d} r^{2}}{w(r)^{2}}-r^{2}\left(\mathrm{~d} \theta^{2}+\sin ^{2} \theta \mathrm{d} \phi^{2}\right),
\end{aligned}
$$

defined on the radial domain $D_{r}=\left(r_{+}, \infty\right)$ where

$w(r)=\left[1-\frac{2 M}{r}+\frac{Q^{2}}{r^{2}}\right]^{\frac{1}{2}}=\left(1-\frac{r_{+}}{r}\right)^{\frac{1}{2}}\left(1-\frac{r_{-}}{r}\right)^{\frac{1}{2}}$,

and $r_{ \pm}=M \pm \sqrt{M^{2}-Q^{2}}$ provided $Q<M$. The corresponding Coulomb potential gives the potential energy,

$$
\frac{Q}{r} \rightarrow V(r)=\frac{e Q}{r},
$$

of the fermion carrying the elementary electric charge $e=$ $\pm \sqrt{\alpha}{ }^{1}$ In the following we study the scattering solutions of the Dirac equation in the asymptotic domain where $r \gg r_{+}$.

We have shown $[17,19]$ that in the gauge we consider here the spherical variables of the Dirac equation can be separated just as in the case of the central problems in Minkowski spacetime [20]. Consequently, the Dirac equation can be put in Hamiltonian form, $H_{D} \tilde{\psi}=i \partial_{t} \tilde{\psi}$ where $\tilde{\psi}=r w(r)^{\frac{1}{2}}$ and the Hamiltonian operator [19]

$$
\begin{aligned}
H_{D}= & -i \frac{w(r)^{2}}{r^{2}}\left(\gamma^{0} \gamma^{i} x^{i}\right)\left(1+x^{i} \partial_{i}\right)-i \frac{w(r)}{r^{2}}\left(\gamma^{i} x^{i}\right) K \\
& +w(r) \gamma^{0} m+V(r),
\end{aligned}
$$

depends on the Dirac angular operator $K=\gamma^{0}(2 \mathbf{S} \cdot \mathbf{L}+1)$, which is associated with the angular variables. Then the particle-like solutions of energy $E$ are the common eigenspinors of the operators $\left\{H_{D}, K, J_{3}\right\}$ corresponding to the eigenvalues $\left\{E, \kappa, m_{j}\right\}$,

$$
\begin{aligned}
& U_{E, \kappa, m_{j}}(x)=U_{E, \kappa, m_{j}}(t, r, \theta, \phi)=\frac{e^{-i E t}}{r w(r)^{\frac{1}{2}}} \\
& \times\left[f_{E, \kappa}^{+}(r) \Phi_{m_{j}, \kappa}^{+}(\theta, \phi)+f_{E, \kappa}^{-}(r) \Phi_{m_{j}, \kappa}^{-}(\theta, \phi)\right],
\end{aligned}
$$

\footnotetext{
${ }^{1}$ In this system $\alpha \simeq \frac{1}{137}$ is the fine structure constant while the electron mass is $m_{e}=\sqrt{\alpha_{G}} \simeq 4.17810^{-23}$.
}

expressed in terms of radial wave functions, $f_{E, K}^{ \pm}$, and the usual four-component angular spinors $\Phi_{m_{j}, \kappa}^{ \pm}$[20]. These spinors are orthogonal to each other, and they are labeled by the angular quantum numbers $m_{j}$ and

$\kappa= \begin{cases}j+\frac{1}{2}=l & \text { for } \quad j=l-\frac{1}{2}, \\ -\left(j+\frac{1}{2}\right)=-l-1 & \text { for } \quad j=l+\frac{1}{2},\end{cases}$

which encapsulates the information as regards the quantum numbers $l$ and $j=l \pm \frac{1}{2}$ as defined in Refs. [20,21] (while in Ref. [7] $\kappa$ is of opposite sign). We note that the antiparticle-like energy eigenspinors can be obtained directly using charge conjugation as in the flat case [22].

Thus the problem of the angular motion is completely solved as in special relativity and we can proceed as in Ref. [20] for deriving the pair of radial equations we need for determining the radial functions, $f^{ \pm}$(denoted from now on without indices). These can be seen as the components of the two-component vectors, $\mathcal{F}=\left(f^{+}, f^{-}\right)^{T}$, while the radial equations can be written as the eigenvalue problem $H_{r} \mathcal{F}=$ $E \mathcal{F}$ of the radial Hamiltonian [19],

$H_{r}=\left(\begin{array}{ll}m w(r)+V(r) & -w(r)^{2} \frac{\mathrm{d}}{\mathrm{d} r}+\frac{\kappa}{r} w(r) \\ w(r)^{2} \frac{\mathrm{d}}{\mathrm{d} r}+\frac{\kappa}{r} w(r)-m w(r)+V(r)\end{array}\right)$

Notice that the standard relativistic scalar product of the Dirac theory [19] now reduces to the radial scalar product

$\left(\mathcal{F}, \mathcal{F}^{\prime}\right)=\left\langle U, U^{\prime}\right\rangle=\int_{D_{r}} \frac{\mathrm{d} r}{w(r)^{2}} \mathcal{F}^{\dagger} \mathcal{F}^{\prime}$,

since the angular spinors are orthonormalized with respect to their own angular scalar product [20].

The resulting radial problem cannot be solved analytically, such that we are forced to resort to the same method of approximation as in Ref. [8] by using a convenient Novikov dimensionless coordinate $[23,24]$. In the present case we chose the Novikov coordinate corresponding to the event horizon of radius $r_{+}$defined as

$x=\sqrt{\frac{r}{r_{+}}-1} \in(0, \infty)$.

Then, by changing the variable, multiplying by $x^{-1}\left(1+x^{2}\right)$ and introducing the notations

$\mu=r_{+} m, \quad \varepsilon=r_{+} E, \quad \delta=\sqrt{\frac{r_{-}}{r_{+}}}$, 
we rewrite the exact radial problem in the form $\mathcal{E F}=0$ with the new matrix operator (15)

$\mathcal{E}=\left(\begin{array}{ll}A & B \\ C & D\end{array}\right)$

with $A, B, C, D$ given by

$A=\mu \sqrt{1+x^{2}-\delta^{2}}-\varepsilon\left(x+\frac{1}{x}\right)+\frac{e Q}{x}$,

$B=-\frac{1}{2} \frac{1+x^{2}-\delta^{2}}{1+x^{2}} \frac{\mathrm{d}}{\mathrm{d} x}+\frac{\kappa \sqrt{1+x^{2}-\delta^{2}}}{1+x^{2}}$

$C=\frac{1}{2} \frac{1+x^{2}-\delta^{2}}{1+x^{2}} \frac{\mathrm{d}}{\mathrm{d} x}+\frac{\kappa \sqrt{1+x^{2}-\delta^{2}}}{1+x^{2}}$,

$D=-\mu \sqrt{1+x^{2}-\delta^{2}}-\varepsilon\left(x+\frac{1}{x}\right)+\frac{e Q}{x}$,

suitable for further approximations.

For very large values of $x$, we can use the Taylor expansion of these equations with respect to $\frac{1}{x}$, neglecting the terms of the order $O\left(1 / x^{2}\right)$. We obtain thus the asymptotic radial problem $[8,25]$, which can be rewritten as $\mathcal{E}^{\prime} \mathcal{F}=0$ where the new matrix operator takes the form

$\mathcal{E}^{\prime}=\left(\begin{array}{ll}\frac{1}{2} \frac{\mathrm{d}}{\mathrm{d} x}+\frac{\kappa}{x} & -x(\mu+\varepsilon)-\frac{1}{x}(\zeta+\beta) \\ x(\varepsilon-\mu)-\frac{1}{x}(\zeta-\beta) & \frac{1}{2} \frac{\mathrm{d}}{\mathrm{d} x}-\frac{\kappa}{x}\end{array}\right)$,

after reversing between themselves the lines and introducing the notations

$\zeta=\frac{m}{2}\left(r_{+}-r_{-}\right)=\frac{1}{2} \mu\left(1-\delta^{2}\right)$

$\beta=\varepsilon-e Q=\varepsilon-e \delta r_{+}$.

As in the Dirac-Coulomb case [21] it is useful to put in diagonal form the terms proportional to $x$ by using the matrix

$T=\left(\begin{array}{ll}-i \sqrt{\mu+\varepsilon} & i \sqrt{\mu+\varepsilon} \\ \sqrt{\varepsilon-\mu} & \sqrt{\varepsilon-\mu}\end{array}\right)$,

for transforming the radial doublet as $\mathcal{F} \rightarrow \hat{\mathcal{F}}=T^{-1} \mathcal{F}=$ $\left(\hat{f}^{+}, \hat{f}^{-}\right)^{T}$. Then we obtain the new system of radial equations

$$
\frac{x}{2} \frac{\mathrm{d} \hat{f}^{ \pm}}{\mathrm{d} x} \pm i\left(\frac{\zeta \mu-\beta \varepsilon}{v}-v x^{2}\right) \hat{f}^{ \pm}=\left(\kappa \pm i \frac{\zeta \varepsilon-\beta \mu}{v}\right) \hat{f}^{\mp}
$$

where $v=\sqrt{\varepsilon^{2}-\mu^{2}}$. These equations can be solved analytically for any values of $\varepsilon$, but here we restrict ourselves to the scattering modes corresponding to the continuous spectrum $\varepsilon \in[\mu, \infty)$. These solutions can be expressed in terms of Whittaker functions as $[8,25]$

$\hat{f}^{+}(x)=C_{1}^{+} \frac{1}{x} M_{\rho_{+}, s}\left(2 i v x^{2}\right)+C_{2}^{+} \frac{1}{x} W_{\rho_{+}, s}\left(2 i v x^{2}\right)$,
$\hat{f}^{-}(x)=C_{1}^{-} \frac{1}{x} M_{\rho_{-}, s}\left(2 i v x^{2}\right)+C_{2}^{-} \frac{1}{x} W_{\rho_{-}, s}\left(2 i v x^{2}\right)$,

where we denote

$s=\sqrt{\kappa^{2}+\zeta^{2}-\beta^{2}}, \quad \rho_{ \pm}=\mp \frac{1}{2}-i q, \quad q=\frac{\beta \varepsilon-\zeta \mu}{v}$.

The integration constants must satisfy [25]

$\frac{C_{1}^{-}}{C_{1}^{+}}=\frac{s-i q}{\kappa-i \lambda}, \quad \frac{C_{2}^{-}}{C_{2}^{+}}=-\frac{1}{\kappa-i \lambda}, \quad \lambda=\frac{\beta \mu-\zeta \varepsilon}{\nu}$.

We observe that the functions $M_{\rho_{ \pm}, s}\left(2 i v x^{2}\right)=\left(2 i v x^{2}\right)^{s+\frac{1}{2}}$ $\left[1+O\left(x^{2}\right)\right]$ are regular in $x=0$, where the functions $W_{\rho_{ \pm}, s}\left(2 i v x^{2}\right)$ diverge as $x^{1-2 s}$ if $s>\frac{1}{2}$ [26]. These solutions will help us to find the scattering amplitudes of the Dirac particles by charged black holes, after fixing the integration constants.

\section{Partial wave analysis}

The scattering of Dirac fermions on a charged black hole is described by the energy eigenspinor $U$ whose asymptotic form,

$U \rightarrow U_{\text {plane }}(\mathbf{p})+A(\mathbf{p}, \mathbf{n}) U_{\mathrm{sph}}$,

for $r \rightarrow \infty$ (where the gravitational and Coulomb fields vanish) is given by the plane wave spinor of momentum $\mathbf{p}$, the free spherical spinors of the flat case behaving as

$U_{\mathrm{sph}} \propto \frac{1}{r} e^{i p r-i E t}, \quad p=\sqrt{E^{2}-m^{2}}=\frac{v}{r_{+}}$,

since in the asymptotic zone the fermion energy is that of special relativity, $E=\sqrt{m^{2}+p^{2}}$. Here we fix the geometry such that $\mathbf{p}=p \mathbf{e}_{3}$, while the direction of the scattered fermion is given by the scattering angles $\theta$ and $\phi$, which are just the spheric angles of the unit vector $\mathbf{n}$. Then the scattering amplitude

$A(\mathbf{p}, \mathbf{n})=f(\theta)+i g(\theta) \frac{\mathbf{p} \wedge \mathbf{n}}{|\mathbf{p} \wedge \mathbf{n}|} \cdot \boldsymbol{\sigma}$ 
depends on the two scalar amplitudes, $f(\theta)$ and $g(\theta)$, which can be studied by using partial wave analysis.

\subsection{Phase shifts}

The partial wave analysis exploits the asymptotic form of the exact analytic solutions which satisfy suitable boundary conditions that in our case might be fixed at the (exterior) event horizon (where $x=0$ ). Unfortunately, here we have only the asymptotic solutions (21) and (22) whose integration constants cannot be related to those of the solutions near the event horizon without resorting to numerical methods [1-7]. Therefore, we must choose suitable asymptotic conditions for determining the integration constants.

The arguments presented in Appendix C of Ref. [8] and briefly summarized here in Appendix A show that for $Q=0$ we may obtain elastic collisions, with real phase shifts and a correct Newtonian limit for large angular momenta, only if we adopt the general asymptotic condition $C_{2}^{+}=C_{2}^{-}=0$, eliminating thus the terms that diverge for $x \rightarrow 0$. We believe that it is natural to keep this condition in the most general case of the Reissner-Nordström metric if we desire to have a smooth limit for $Q \rightarrow 0$.

The asymptotic form of the doublet $\mathcal{F}=T \hat{\mathcal{F}}$ can be obtained as in Ref. [8] observing that now we must replace $v x^{2}=p\left(r-r_{+}\right)$. Thus we obtain the definitive asymptotic form of the radial functions of the scattered fermions by charged black holes,

$$
\begin{aligned}
\mathcal{F} & =\left(\begin{array}{l}
i \sqrt{\varepsilon+\mu}\left(\hat{f}^{-}-\hat{f}^{+}\right) \\
\sqrt{\varepsilon-\mu}\left(\hat{f}^{+}+\hat{f}^{-}\right)
\end{array}\right) \\
& \propto \sqrt{E+m} \sin \left(p r-\frac{\pi l}{2}+\delta_{\kappa}+\vartheta(r)\right),
\end{aligned}
$$

whose point-independent phase shifts $\delta_{\kappa}$ give the quantities

$$
S_{\kappa}=e^{2 i \delta_{\kappa}}=\left(\frac{\kappa-i \lambda}{s-i q}\right) \frac{\Gamma(1+s-i q)}{\Gamma(1+s+i q)} e^{i \pi(l-s)} .
$$

Notice that the values of $\kappa$ and $l$ are related as in Eq. (10), i.e. $l=|\kappa|-\frac{1}{2}(1-\operatorname{sign} \kappa)$. The remaining point-dependent phase,

$\vartheta(r)=-p r_{+}+q \ln \left[2 p\left(r-r_{+}\right)\right]$,

which does not depend on angular quantum numbers, may be ignored as in the Dirac-Coulomb case [7,21].

We arrived thus at the final result (29) depending on the parameters introduced above that can be expressed in terms of physical quantities $m, M, e, Q \ldots$ etc. In addition, assuming that the inequality $|\beta| \geq|\zeta|$ holds even for $p=0$, it is convenient to introduce the new real parameter $k$ obeying

$$
k^{2}=\beta^{2}-\zeta^{2}=q^{2}-\lambda^{2},
$$

which allows us to write simply $s=\sqrt{\kappa^{2}-k^{2}}$. Then by using Eq. (26b) we obtain our principal new result, which holds for massive fermions:

$$
\begin{aligned}
k & =\left[\left(r_{+} E-e Q\right)^{2}-\frac{m^{2}}{4}\left(r_{+}-r_{-}\right)^{2}\right]^{\frac{1}{2}}, \\
q & =r_{+} p+M \frac{m^{2}}{p}-e Q \frac{E}{p}, \\
\lambda & =m M \frac{E}{p}-e Q \frac{m}{p} .
\end{aligned}
$$

Obviously, in the case of the massless neutral fermions (with $m=e=0$ ) we are left with the unique parameter $q=r_{+} p$ since then $\lambda=0$ and $k=q$. Hereby we see that the parameter $s$ has a special role to play since it can take either real values or pure imaginary ones regardless of the fermion mass.

\subsection{Elastic scattering}

For the real values of $s$ the scattering is elastic since in this case the identity (31) guarantees that the phase shifts of Eq. (29) are real numbers such that $\left|S_{K}\right|=1$. Obviously, this happens only when $\kappa$ (at given $p$ ) satisfies the condition

$|\kappa| \geq \tilde{k}+1$,

where $\tilde{k}=$ floor $(k)$ is the greatest integer less than $k$. Then the scalar amplitudes of Eq. (27),

$f(\theta)=\sum_{l=0}^{\infty} a_{l} P_{l}(\cos \theta), \quad g(\theta)=\sum_{l=1}^{\infty} b_{l} P_{l}^{1}(\cos \theta)$,

depend on the following partial amplitudes [7,21]:

$$
\begin{aligned}
& a_{l}=(2 l+1) f_{l}=\frac{1}{2 i p}\left[(l+1)\left(S_{-l-1}-1\right)+l\left(S_{l}-1\right)\right], \\
& b_{l}=(2 l+1) g_{l}=\frac{1}{2 i p}\left(S_{-l-1}-S_{l}\right),
\end{aligned}
$$

and they give rise to the elastic scattering intensity or differential cross section,

$\mathcal{I}(\theta)=\frac{\mathrm{d} \sigma}{\mathrm{d} \Omega}=|f(\theta)|^{2}+|g(\theta)|^{2}$,

and the polarization degree,

$\mathcal{P}(\theta)=-i \frac{f(\theta)^{*} g(\theta)-f(\theta) g(\theta)^{*}}{|f(\theta)|^{2}+|g(\theta)|^{2}}$.

This last quantity is interesting for the scattering of massive fermions representing the induced polarization for an unpolarized initial beam. 


\subsection{Absorption}

The absorption is present in the partial waves for which we have

$$
1 \leq|\kappa| \leq \tilde{k}
$$

Here we meet a branch point in $s=0$ and two solutions $s= \pm i|s|= \pm i \sqrt{k^{2}-\kappa^{2}}$, between them we must chose $s=-i|s|$ since only in this manner we select the physical case of $\left|S_{\kappa}\right|<1$. More specific, by substituting $s=-i|s|$ in Eq. (29) we obtain the simple closed form

$\left|S_{\kappa}\right|=\left|S_{-\kappa}\right|=e^{-2 \Im \delta_{\kappa}}=e^{-\pi|s|} \sqrt{\frac{\sinh \pi(q-|s|)}{\sinh \pi(q+|s|)}}$,

showing that $0<\left|S_{\kappa}\right|<1$ since $|s|<q$ for any $(p, \kappa)$ obeying the condition (40). Moreover, we can verify that in the limit of large momentum (or energy) the absorption tends to become maximal since

$\lim _{p \rightarrow \infty}\left|S_{K}\right|=0$,

regardless of the fermion mass.

Under such circumstances we can calculate the absorption cross section that reads [7]

$\sigma_{a}=\sum_{l \geq 1} \sigma_{a}^{l}(p)=\frac{2 \pi}{p^{2}} \sum_{l=1}^{\tilde{k}} l\left(1-\left|S_{l}\right|^{2}\right)$,

since for $s=-i|s|$ we have $\left|S_{-\kappa}\right|=\left|S_{\kappa}\right|$ as in Eq. (41). This cross section can be calculated at any time as a finite sum of the partial cross sections whose definitive closed form,

$$
\begin{aligned}
\sigma_{a}^{l}(p)= & \theta(k-l) \frac{2 \pi l}{p^{2}} \\
& \times\left[1-e^{-2 \pi \sqrt{k^{2}-l^{2}}} \frac{\sinh \pi\left(q-\sqrt{k^{2}-l^{2}}\right)}{\sinh \pi\left(q+\sqrt{k^{2}-l^{2}}\right)}\right],
\end{aligned}
$$

is derived according to Eqs. (41) and (43), while the condition (40) introduces the Heaviside step function $\theta(k-l)$. Hereby we understand that absorption arises in the case of the partial wave $l$ for the values of $p$ (or $E$ ) satisfying the condition $k>l$. This means that for any fixed value of $l$ there is a threshold, $E_{l}$, defined as the positive solution of the equation

$$
\left|r_{+} E_{l}-e Q\right|=\sqrt{l^{2}+\frac{1}{4} m^{2}\left(r_{+}-r_{-}\right)^{2}} .
$$

A similar condition with (45) was obtained in Ref. [27] for absorption on a dilaton black hole. This indicates that the fermions with $|\kappa|=l$ can be absorbed by a black hole only if $E \geq E_{l}$. The existence of these thresholds is important since these keep under control the effect of the singularities in $p=0$.

Finally, we observe that in the high-energy limit all these absorption cross sections tend to the event horizon (apparent) area, indifferent on the fermion mass $m \geq 0$, as it results from Eq. (42), which yields

$\lim _{p \rightarrow \infty} \sigma_{a}=\lim _{p \rightarrow \infty} \frac{2 \pi}{p^{2}} \sum_{l=1}^{\tilde{k}} 1=\lim _{p \rightarrow \infty} \frac{\pi}{p^{2}} \tilde{k}(\tilde{k}+1)=\pi r_{+}^{2}$,

since $\tilde{k}(\tilde{k}+1) \sim k^{2} \sim r_{+}^{2} p^{2}$.

\section{Graphical discussion of the results}

Let us now discuss some physical consequences of our results encapsulated by the formulas presented in the previous sections. For a better understanding of the analytical results we perform a graphical analysis of the differential cross section in terms of the scattering angle $\theta$.

All the plots are obtained using the methods described in Ref. [8] where we used a technique proposed some time ago by Yennie et al. [28], which consists of replacing the series from Eq. (36) by the $m$ th reduced ones,

$$
\begin{aligned}
& f(\theta)=\frac{1}{(1-\cos \theta)^{m_{1}}} \sum_{l \geq 0} a_{l}^{\left(m_{1}\right)} P_{l}(\cos \theta), \\
& g(\theta)=\frac{1}{(1-\cos \theta)^{m_{2}}} \sum_{l \geq 1} b_{l}^{\left(m_{2}\right)} P_{l}^{1}(\cos \theta) .
\end{aligned}
$$

The recurrence relations satisfied by the Legendre polynomials $P_{l}(x), P_{l}^{1}(x)$ lead to the iterative rules giving the reduced coefficients in any order

$$
\begin{aligned}
& a_{l}^{(i+1)}=a_{l}^{(i)}-\frac{l+1}{2 l+3} a_{l+1}^{(i)}-\frac{l}{2 l-1} a_{l-1}^{(i)}, \\
& b_{l}^{(i+1)}=b_{l}^{(i)}-\frac{l+2}{2 l+3} b_{l+1}^{(i)}-\frac{l-1}{2 l-1} b_{l-1}^{(i)},
\end{aligned}
$$

if we start with $a_{l}^{(0)}=a_{l}$ and $b_{l}^{(0)}=b_{l}$ as defined by Eqs. (37). As in Ref. [8] using the second iteration for $f\left(m_{1}=2\right)$ and the first one for $g\left(m_{2}=1\right)$ seems to be satisfactory without distorting the analytical results.

In the following we focus our analysis on scattering from small or micro black holes (with $M \sim 10^{15}-10^{22} \mathrm{~kg}$ ) since in this case the wave length of the fermion $(\lambda=2 \pi h / p)$ and the Schwarszchild radius $\left(r_{S}=2 M\right)$ have the same order of magnitude so that we can observe the presence of glory and orbiting scattering. 


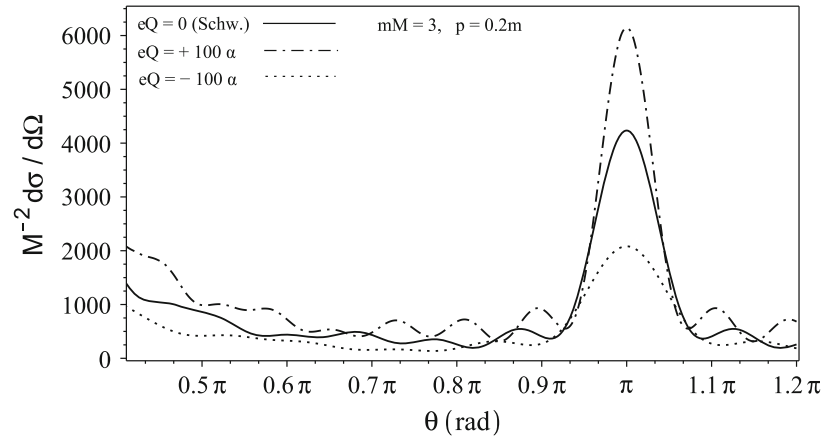

Fig. 1 Comparison between the scattering cross section of ReissnerNordström black hole and the Schwarzschild black hole for $p=0.2 \mathrm{~m}$ and $m M=3$

Comparing the scattering cross section of a ReissnerNordström black hole with that of a Schwarzschild black hole (see Fig. 1) one can observe several things. First is that in the case of scattering by the Reissner-Nordström black hole the differential cross section will increase if the charge of the black hole and the incoming fermions charge have the same sign (the dash-dotted line in Fig. 1). Thus we can say that the electric repulsion increases the scattering cross section as expected, according to (45), comparatively with the scattering on Schwarzschild black hole (the solid line in Fig. 1). In the opposite case, when the black hole charge and the incoming fermions charge have opposite signs, then the electric attraction between the two charges will imply that more fermions are absorbed by the black hole, thus lowering the scattering cross section comparatively with the scattering on Schwarzschild black hole (as can be seen from the dotted line in Fig. 1). Figure 1 also reveals the fact that the width of the glory peak becomes more pronounced when the sign of the total black hole charge is the same as the charge of the fermion, respectively, the glory peak's width decreases if the black hole and the fermions have opposite signs. We can conclude that when the charge of the black hole and fermions charge have the same sign, the glory scattering will be a phenomenon which becomes important comparatively with the Schwarzschild case.

In Figs. 2 and 3 we present the differential cross section in terms of the scattering angle for the Reissner-Nordström black hole. The effects of electric interaction (between the black hole and fermion charges) on the glory and orbiting scattering can also be observed. We have found that if the black hole has opposite charge than the incoming fermion then, as we increase the charge on the black hole, the glory (i.e. backward scattering at angles close to $\pi$ ) starts to decrease up to a point when it will disappear completely (see Fig. 2). The same is true for the orbiting scattering (i.e. scattering for $\theta<\pi$ ) for which we observe a decrease in oscillations as the black hole charge increases. This should not come as a surprise since in this case the opposite signs

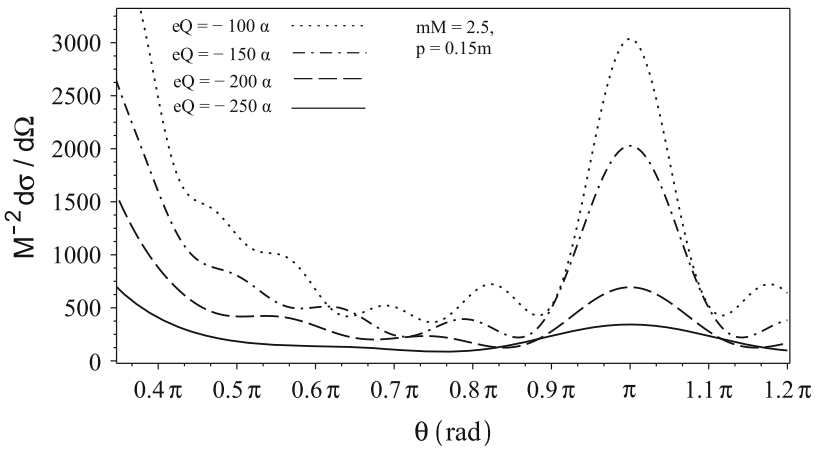

Fig. 2 Reissner-Nordström black hole scattering cross section for $p=$ $0.15 m, m M=2.5$ and different negative values of $e Q$. In all plots $\alpha \approx 1 / 137$ represents the fine structure constant

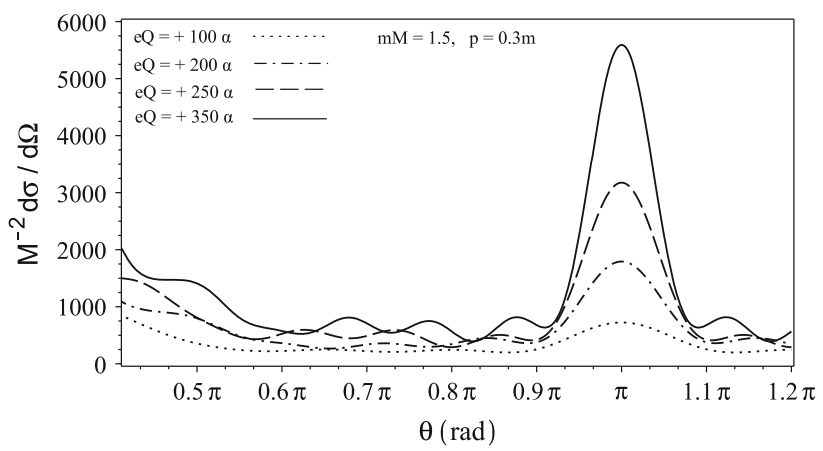

Fig. 3 Reissner-Nordström black hole scattering cross section for $p=$ $0.3 m, m M=1.5$ and different positive values of $e Q$

of the charges will increase the absorption in the black hole. On the contrary if the black hole and fermion's charges have the same sign (see Fig. 3), then the glory peak will increase as the black hole becomes more charged. The same is true for the orbiting scattering for which the oscillatory behavior becomes more pronounced as we add more charge on the black hole. Regarding the forward scattering, we have found it to be divergent for $\theta \rightarrow 0$ as can be seen from Fig. 2. This is in fact an effect of the long range nature of the gravitational and electric potentials, which both behave as $1 / r$.

Regarding the effects of the black hole's charge on the induced polarization after the scattering of an initially unpolarized beam we can say according to Fig. 4 the following: (i) compared with the Schwarzschild polarization (dotted line) the Reissner-Nordström polarization is a less oscillating function if the black hole has opposite charge that the incoming fermions (dashed line), respectively, the oscillations become more fervent if the black hole charge has the same sign as the incoming fermions (solid line); (ii) the oscillations appearing in the polarization can be seen as resulting from the oscillatory behavior of glory/orbiting scattering as well as from the forward/backward one.

The dependence of the absorption cross section in terms of energy $(E / m)$ is given in Fig. 5, where the plots were 


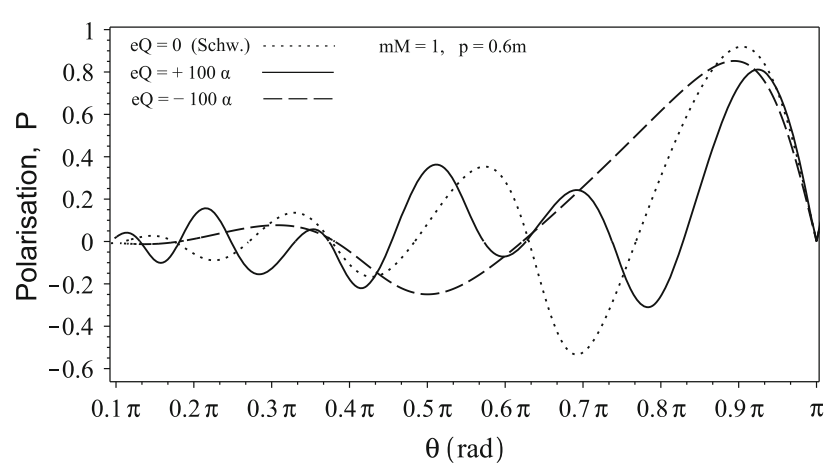

Fig. 4 Comparison of the Schwarzschild polarization (dotted line) with Reissner-Nordström polarization (dashed line for positive charge on the black hole, respectively solid line for negative charge on the black hole) for $p=0.6 m$ and $m M=1$

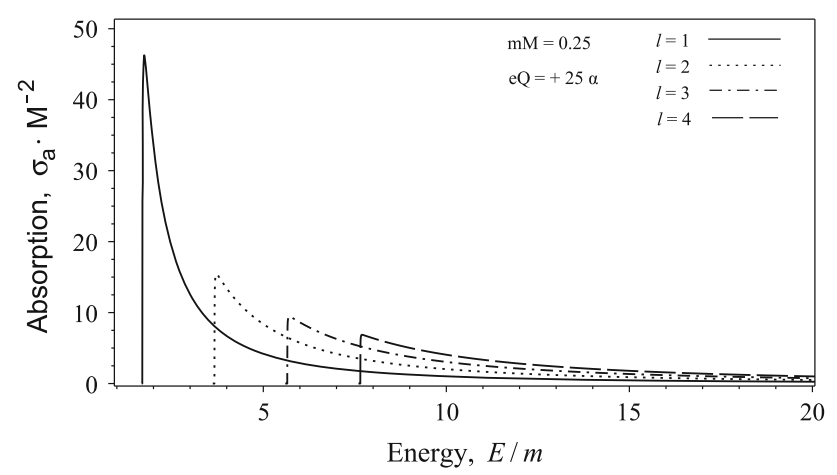

Fig. 5 Partial absorption cross section as a function of $E / m$ for the Reissner-Nordström black hole with $e Q=0.25 \alpha$ and $m M=0.25$, for given values of angular momentum $l=1,2,3,4$

obtained for $l=1,2,3,4$. We observe that the modes with small angular momenta have the most important contribution to the absorption, because as we increase $l$ the maxima observed in Fig. 5 becomes smaller. Also we observe that the maxima are shifted to the right as we increase the value of $l$. Our graphical result for the absorption cross section is similar to those obtained in [27], where the absorption of scalar particles on a dilaton black hole was studied.

\section{Concluding remarks}

This is the basic framework of the relativistic partial wave analysis of the Dirac fermions scattered by charged black holes in which we consider exclusively the contribution of the scattering modes. Our results are in accordance with the Newtonian limit since in the large- $l$ limits and for very small momentum we can take $s \sim|\kappa| \sim l$ and $\lambda \sim q$ so that our phase shifts (29) become just the Newtonian ones [1,2].

The above results encapsulate a new interesting particular case, namely, Dirac-Coulomb scattering in the presence of the gravitational field of the charged target that gives rise to a Schwarzschild gravitational field instead of a ReissnerNordström one. In this situation we must take $\delta \rightarrow 0$ everywhere apart from the Coulomb term so that $r_{+} \rightarrow 2 M$ and $r_{-} \rightarrow 0$. Thus we are left with the partial waves given by Eq. (29) but with the new parameters

$$
\begin{aligned}
k & \rightarrow\left[(2 M E-e Q)^{2}-m^{2} M^{2}\right]^{\frac{1}{2}} \\
q & \rightarrow 2 M p+M \frac{m^{2}}{p}-e Q \frac{E}{p} \\
\lambda & \rightarrow m M \frac{E}{p}-e Q \frac{m}{p} .
\end{aligned}
$$

Now we observe that for $Q=0$ we recover the results of Ref. [8] concerning the collision between a Dirac fermion and a Schwarzschild (neutral) black hole. Moreover, if we keep $Q \neq 0$ taking $M \rightarrow 0$ we recover the well-known DiracCoulomb scattering in Minkowski spacetime [21] with the parameters

$k \rightarrow|e Q|, \quad q \rightarrow-e Q \frac{E}{p}, \quad \lambda \rightarrow-e Q \frac{m}{p}$.

The conclusion is that here we derived the most general results of the Dirac-Coulomb scattering in central gravitational fields.

Acknowledgments C.A. Sporea was supported by a grant of the Romanian National Authority for Scientific Research, Programme for research-Space Technology and Advanced Research-STAR, project nr. 72/29.11.2013 between Romanian Space Agency and West University of Timisoara.

Open Access This article is distributed under the terms of the Creative Commons Attribution 4.0 International License (http://creativecomm ons.org/licenses/by/4.0/), which permits unrestricted use, distribution, and reproduction in any medium, provided you give appropriate credit to the original author(s) and the source, provide a link to the Creative Commons license, and indicate if changes were made.

Funded by $\mathrm{SCOAP}^{3}$.

\section{Appendix A: Condition of elastic scattering}

Let us briefly present the main arguments for choosing the asymptotic conditions $C_{2}^{+}=C_{2}^{-}=0$ in deriving the phase shifts (29). It is convenient to introduce the new notations (up to a real arbitrary common factor)

$C_{1}^{+}=e^{i \theta_{1}}, \quad C_{2}^{+}=C e^{i \theta_{2}}$,

$C_{1}^{-}=e^{\theta_{1}} \frac{s-i q}{\kappa-i \lambda}, \quad C_{2}^{-}=-\frac{C}{\kappa-i \lambda} e^{i \theta_{2}}$,

where $C, \theta_{1}$, and $\theta_{2}$ are real-valued parameters. Then, according to Eq. $\mathrm{C} 2$ from Appendix C in Ref. [8], the general expres- 
sion of the phase shifts reads

$$
e^{2 i \delta_{\kappa}}=\frac{\kappa-i \lambda}{s+i q} \frac{\frac{\Gamma(1+2 s)}{\Gamma(s+i q)} e^{i \pi(l-s)}}{\frac{\Gamma(1+2 s)}{\Gamma(s-i q)}-C e^{i \theta} e^{-i \pi(s+i q)}} .
$$

We observe that the phase shifts depend only on two real integration constants, $C$, and the relative phase $\theta=\theta_{1}-\theta_{2}$.

The elastic scattering can arise only when we have $\left|e^{2 i \delta_{\kappa}}\right|=1$. There are two cases. In the first one, when $s=|s|$ is a pure real number, the equation $\left|e^{2 i \delta_{\kappa}}\right|=1$ has two real solutions, $C=0$ and

$$
\begin{aligned}
C= & e^{-\pi q} \frac{\Gamma(1+2 s)}{|\Gamma(s+i q)|^{2}} \\
& \times\left[e^{i(\pi s-\theta)} \Gamma(s+i q)+e^{-i(\pi s-\theta)} \Gamma(s-i q)\right] .
\end{aligned}
$$

In the second case when $s= \pm i|s|$ is a pure imaginary number, the equation $\left|e^{2 i \delta_{\kappa}}\right|=1$ has no real solutions.

It can easily be showed (for details see Ref. [8]) that for $Q=0$ and large values of $l$ the phase shifts A.2 have a correct Newtonian limit

$e^{2 i \delta_{l}^{N}}=\frac{\Gamma(1+l-i q)}{\Gamma(1+l+i q)}, \quad q=2 M p+\frac{M m^{2}}{p}$

only if we chose the asymptotic condition $C=0$ (i.e. $C_{2}^{+}=$ $C_{2}^{-}=0$ ). Otherwise, if we consider the solution (A.3) we obtain non-determinate phase shifts

$e^{2 i \delta_{\kappa}}=-\frac{\kappa-i \lambda}{s+i q} e^{i(\pi l+\pi s-2 \theta)}$,

which still depend on the arbitrary phase $\theta$. Obviously, in this case we cannot talk about the Newtonian limit.
4. J. Jing, Phys. Rev. D 71, 124006 (2005)

5. K.H.C. Castello-Branco, R.A. Konoplya, A. Zhidenko, Phys. Rev. D 71, 047502 (2005)

6. C. Doran, A. Lasenby, S. Dolan, I. Hinder, Phys. Rev. D 71, 124020 (2005)

7. S. Dolan, C. Doran, A. Lasenby, Phys. Rev. D 74, 064005 (2006)

8. I.I. Cotăescu, C. Crucean, C.A. Sporea, Eur. Phys. J. C 76, 102 (2016). arXiv:1409.7201

9. C.L. Benone, E.S. Oliveira, S.R. Dolan, L.C.B. Crispino, Phys. Rev. D 89, 104053 (2014)

10. L.C.B. Crispino, S.R. Dolan, E.S. Oliveira, Phys. Rev. D 79, 064022 (2009)

11. C.F.B. Macedo, L.C.B. Crispino, Phys. Rev. D 90, 064001 (2014)

12. R.A. Konoplya, A. Zhidenko, Phys. Rev. D 76, 084018 (2007)

13. L.C.B. Crispino, S.R. Dolan, E.S. Oliveira, Phys. Rev. Lett. 102, $231103(2009)$

14. L.C.B. Crispino, S.R. Dolan, A. Higuchi, E.S. Oliveira, Phys. Rev. D 90, 064027 (2014)

15. L.C.B. Crispino, S.R. Dolan, A. Higuchi, E.S. Oliveira, Phys. Rev. D 92, 084056 (2015)

16. E.S. Oliveira, L.C.B. Crispino, A. Higuchi, Phys. Rev. D 84, 084048 (2011)

17. I.I. Cotăescu, Mod. Phys. Lett. A 13, 2923 (1998)

18. I.I. Cotăescu, J. Phys. A Math. Gen. 33, 1977 (2000)

19. I.I. Cotăescu, Roman. J. Phys. 52, 895 (2007)

20. B. Thaller, The Dirac Equation (Springer Verlag, Berlin, Heidelberg, 1992)

21. V.B. Berestetski, E.M. Lifshitz, L.P. Pitaevski, Quantum Electrodynamics (Pergamon Press, Oxford, 1982)

22. I.I. Cotăescu, Phys. Rev. D 60, 124006 (1999)

23. I.D. Novikov, doctoral disertation, Sthernberg Astronomical Institute (1963)

24. C.W. Misner, K.S. Thorne, J.A. Wheeler, Gravitation (Freeman \& Co., San Francisco, 1971)

25. I.I. Cotăescu, Mod. Phys. Lett. A 22, 2493 (2007)

26. F.W.J. Olver, D.W. Lozier, R.F. Boisvert, C.W. Clark, NIST Handbook of Mathematical Functions (Cambridge University Press, 2010)

27. I. Sakalli, O.A. Aslan, Astropart. Phys. 74, 73-78 (2016)

28. D.R. Yennie, D.G. Ravenhall, R.N. Wilson, Phys. Rev. 95, 500 (1954)

\section{References}

1. J.A.H. Futterman, F.A. Handler, R.A. Matzner, Scattering from Black Holes (Cambridge University Press, Cambridge, 1988)

2. N.K. Kofiniti, Int. J. Theor. Phys. 23, 991 (1984)

3. J. Jing, Phys. Rev. D 70, 065004 (2004) 